\title{
Tratamento da espondilólise sintomática com reparo direto pela técnica de Buck modificada
}

\author{
Modified Buck's direct repair to simptomatic spondylolysis treatment \\ Tratamiento de la espondilolisis sintomática con preparo directo por la \\ técnica de Buck modificada
}

\author{
Bruno Pinto Coelho Fontes' \\ Maurício Erwin Pühler Resende ${ }^{2}$ \\ Carlos Moreira Rezende' \\ Rodrigo D'Alessandro de Macedo ${ }^{3}$ \\ Luis Olímpio Garcia Pedrosa ${ }^{4}$ \\ Jefferson Soares Leal ${ }^{5}$ \\ Rogério Lúcio Chaves de Resende ${ }^{5}$
}

\section{RESUMO}

Objetivo: justificar a reconstrução da pars interarticularis pela técnica modificada de Buck como método efetivo no tratamento de lombalgia por espondilólise ou espondilolistese tipo 1, refratárias ao tratamento conservador. Métodos: avaliação retrospectiva de oito pacientes com idade entre $11 \mathrm{e}$ 34 anos, operados e acompanhados no período de outubro de 2004 a maio de 2008 com a técnica de Buck utilizando-se parafusos canulados de titânio. Nenhum paciente foi imobilizado no pós-operatório. Os pacientes foram selecionados após serem submetidos a estudos por imagem utilizando radiografias e ressonância magnética. Também foram avaliados no pósoperatório, segundo os critérios de Henderson e quanto à satisfação com o tratamento, assim como o índice de consolidação. Resultados: sete pacientes foram classificados como excelentes, retornando ao nível de prá-

\section{ABSTRACT}

Objective: to justify the modified Buck's technique of pars reconstruction as an effective method to spondylolysis and low grade spondylolisthesis treatment. Methods: eight patients with ages 11 to 34 years were retrospectively evaluated from October 2004 to may 2008. They were operated with Buck's technique using titanium canulated screws. Immobilization wasn't used in any patient in post-operative period. All patients were studied with magnetic resonance imaging. All patients were assessed in post-operative period with Henderson's criteria of functional capacity. Results: seven patients were assessed as excellent. They didn't complain pain and were able to return to former occupation and sportive activities. One patient presented temporary paresthesia and leg weakness, and one patient bilateral pseudoarthrosis that cursed with good grade functional capacity.

\section{RESUMEN}

Objetivo: justificar la reconstrucción de la pars interarticularis por la técnica modificada de Buck como método efectivo en el tratamiento de la lumbalgia por espondilosis o espondilolistesis tipo 1, refractarias al tratamiento conservador. Métodos: evaluación retrospectiva de ocho pacientes con edad entre 11 a 34 años, operados y acompañados en el periodo de Octubre de 2004 hasta Mayo de 2008 con la técnica de Buck usando tornillos canulados de titanio. Ningún paciente fue inmovilizado en el postoperatorio. Los pacientes fueron seleccionados después de ser sometidos a estudios por imagen utilizando radiografias $y$ resonancias magnéticas. También fueron evaluados en el postoperatorio, según los criterios de Henderson y el grado de satisfacción con el tratamiento, así como también el índice de consolidación. Resultados: siete pacientes fueron clasificados como excelentes,

\footnotetext{
Trabalho realizado no Hospital da Baleia, Hospital Governador Israel Pinheiro - IPSEMG, Hospital da Policia Militar de MG, Hospital das Clínicas da UniversidadeFederal de Minas Gerais e Hospital Santo Ivo - Belo Horizonte (MG), Brasil.

Não há conflitos de interesse.

'Assistente do Grupo de Cirurgia da Coluna do Hospital da Baleia e Hospital da Polícia Militar de MG - Belo Horizonte (MG), Brasil.

${ }^{2}$ Assistente do Grupo de Cirurgia da Coluna do Hospital Evangélico e Hospital da Polícia Militar de MG - Belo Horizonte (MG), Brasil.

${ }^{3}$ Assistente do Grupo de Cirurgia da Coluna do Hospital da Baleia e Hospital Governador Israel Pinheiro -IPSEMG- Belo Horizonte (MG), Brasil.

${ }^{4}$ Assistente do Grupo de Cirurgia da Coluna do Hospital da Baleia - Belo Horizonte (MG), Brasil.

${ }^{5}$ Assistente do Grupo de Cirurgia da Coluna do Hospital das Clínicas da UFMG e Instituto Biocor - Belo Horizonte (MG), Brasil.
} 
tica esportiva e laboral prévia. Dentre eles, um evoluiu com parestesia transitória, com recuperação completa. A oitava paciente apresentou uma pseudartrose bilateral, mas com bom resultado do ponto de vista clínico. Todos os pacientes ficaram satisfeitos com o resultado. Conclusão: a reconstrução ístmica com parafusos de titânio atingiu excelentes resultados ao aliar biomecânica estável, dissecção mínima da musculatura, com preservação da anatomia e mobilidade do segmento.
All patients were satisfied with treatment. Conclusion: the isthmic reconstruction using titanium screws allowed excellent results allied with stable biomechanics, minimal injury to back muscles, preservation of anatomy and segmental mobility, and better operated spinal imaging.

retornando al nivel de práctica deportiva y laboral previa. Dentro estos siete pacientes, uno evolucionó con parestesias transitorias, con recuperación completa. La octava paciente presentó una pseudoartrosis bilateral, pero con buen resultado desde el punto de vista clínico. Todos los pacientes quedaron satisfechos con el resultado. Conclusión: la reconstrucción ístmica con tornillos de titanio tuvo excelentes resultados al juntar biomecánica estable, disección mínima de la musculatura, con preservación de la anatomía y modalidad del segmento.

KEYWORDS: Low back pain/ surgery; Spondylolysis; Orthopedic procedures/ methods; Bone screws
Descriptores: Dolor de la región lumbar / cirugía; Espondilólisis; Procedimientos ortopédicos/ métodos; Tornillos óseos

\section{INTRODUÇÃO}

Espondilólise é uma lesão de origem indeterminada, que se acredita ser causada por fratura por estresse na pars interarticularis, que é particularmente vulnerável a forçasrepetitivas de flexão, extensão e rotação1. Define-se pars interarticularis como uma parte do arco neural que fica entre as facetas articulares superior e inferior. Nas vértebras lombares a pars interarticularis foi ainda subdividida, sendo o "pilar lateral" (lateral buttress) o que oferece principal suporte estrutural. O pilar lateral é uma ponte óssea que conecta a borda supero-lateral da faceta inferior com a junção do processo transverso e pedículo. Em estudos com cadáveres foi encontrada maior área do pilar lateral em L1 a L3 (80mm2) e a menor em L5 (15 mm2), sendo esse um fator para maior incidência de fraturas por estresse nessa localização ${ }^{2}$.

Clinicamente, observou-se correlação significativa entre espondilólise e degeneração discal nos níveis adjacentes, caudal e cranial, em maior proporção que o esperado para o processo de envelhecimento natural 3 . Do ponto de vista biomecânico, ocorre desconexão do gancho ósseo posterior, resultando em transmissão inapropriada da carga pelos elementos posteriores. Dessa forma, a carga é principalmente transmitida por meio dos elementos anteriores, que induz à concentração inadequada de forças ${ }^{4}$. Estudo experimental revela, na presença de espondilólise, maior sobrecarga discal em resposta a todos os movimentos do segmento acometido.
No nível L5/S1, especialmente no movimento rotacional, observa-se aumento de até $238 \%$ das forças ${ }^{5}$.

A espondilólise lombar é uma condição comum, sendo que apenas uma pequena porcentagem de casos tornase sintomática. Submetidos ao tratamento conservador, a maioria dos pacientes melhora. Entretanto, nos pacientes com dor persistente, refratária ao tratamento conservador, o tratamento cirúrgico é indicado, o que ocorre em torno de $30 \%$ dos $\operatorname{casos}^{6}$.

Existem diversas opções de tratamento cirúrgico para a doença. Entre as mais consagradas está a realização de artrodese, que pode ser realizada por meio de diversas técnicas que apresentam bons resultados clínicos, mas como visam à fusão como resultado final, ocorre perda completa dos movimentos do segmento da coluna abordado ${ }^{7,8}$. Outras técnicas utilizadas são as cirurgias para reconstrução ístmica, que visam à preservação do movimento e redistribuição adequada de forças entre os elementos posteriores e anteriores da coluna. As técnicas e materiais utilizados variam e com eles a rigidez da reconstrução e sua taxa de sucesso em obter fusão, assim como a taxa de complicações.

Entre as técnicas de reconstrução ístmica podemos citar a técnica de Buck, a de Scott e Scott modificada*, a de Morscher**, a de Songer*** e a de Takahashi****. Entre elas, a técnica de Buck demonstra ser a mais estável e com melhores taxas de fusão e resultados clínicos9. Essa técni-

Scott JHS. The Edinburgh repair of isthmic spondylolysis. J Bone Joint Surg [Br]. 1987;69(3):491.

"Morscher E, Gerber B, Fasel J. Surgical treatment of spondylolisthesis by bone grafting and direct stabilization of spondylolysis by means of a hook screw. Arch Orthop Trauma Surg. 1984;103(3): 175-8.

.".Songer MN, Rovin R. Repair of the pars interarticularis defect with a cable screw construct: a preliminary report. Spine. 1998;23(2):263-9.

".*t Tokuhashi Y, Matsuzaki H. Repair of defects in spondylolysis by segmental pedicular screw hook fixation: a preliminary report. Spine. $1996 ; 21$ (17):2041-5. 
ca apresenta a vantagem de restaurar a anatomia normal, ser minimamente invasiva, promover compressão direta do defeito ósseo e ser intra-segmentar, mantendo, assim, a movimentação normal do segmento ${ }^{10}$.

O objetivo deste trabalho é avaliar os resultados clínicos e radiológicos da técnica de Buck modificada com relação ao índice de consolidação, satisfação do paciente e a capacidade de retorno aos mesmos níveis de atividade prévios ao quadro doloroso. Serão avaliados, de forma retrospectiva, todos os casos operados pela técnica de Buck modificada pelas equipes das quais os autores fazem ou já fizeram parte.

\section{MÉTODOS}

Trata-se de um estudo retrospectivo, composto de uma série de casos, realizado em cinco instituições (Hospital da Baleia, Hospital Governador Israel Pinheiro (IPSEMG), Hospital da Policia Militar de MG, HC-UFMG, e Hospital Santo Ivo) de 24/10/2004 a 20/11/2007, em que seis homens e duas mulheres com idade entre 11 e 34 anos foram tratados de espondilólise e espondilolistese grau I por meio do reparo direto do defeito ístmico (Quadro 1). específicos (Reverse Gantry) para a visualização do defeito na pars, pois pequena parte das lesões se encontra no mesmo plano que o platô vertebralı. Nenhum paciente apresentou sinais de lesão aguda (fratura incompleta e sem separação das margens). Todos os pacientes apresentaram sinais de lesão crônica (fratura completa com separação bem delineada, com arredondamento das corticais), dispensando propedêutica adicional com SPECT-Scan. Os pacientes foram também submetidos a estudo com ressonância magnética para avaliar a presença de degeneração discal ${ }^{12,13}$. Na constatação de discos degenerados, os casos foram submetidos à discografia provocativa, sendo excluídos do estudo aqueles com discografia positiva. No presente estudo, apenas um paciente apresentava alterações degenerativas discais incipientes, com discografia negativa (W.C.S.). A indicação cirúrgica deveu-se à dor lombar persistente, mesmo após três a seis meses de tratamento conservador (repouso, medicação, fisioterapia), associada a imagem radiológica de listese grau 0 ou 1 (Meyerding) e defeito ístmico menor que $3 \mathrm{~mm}$.

A técnica de Buck foi modificada devido à utilização de parafusos de titânio canulados, usualmente utilizados em fixações de fraturas de odontóide por via anterior. Esse implante Descriçáo da populaçáo estudada

$\begin{array}{llllll}\text { Nome } & \text { Data da cirurgia } & \text { Hospital } & \begin{array}{l}\text { Idade na data } \\ \text { da cirurgia }\end{array} & \text { Diagnóstico } & \text { Nível afetado } \\ \text { J. S. } & 24.10 .2004 & \text { IPSEMG } & 18 \text { anos } & \text { Espondilolistese } & \text { L4 } \\ \text { F. J. S. } & 12.07 .2005 & \text { Baleia } & 30 \text { anos } & \text { Espondilolistese } & \text { L5 } \\ \text { A. N. M. } & 23.01 .2006 & \text { HC } & 16 \text { anos } & \text { Espondilolistese } & \text { L5 } \\ \text { P. P. } & 30.08 .2006 & \text { HPM } & 31 \text { anos } & \text { Espondilólise } & \text { L5 } \\ \text { W. C. S. } & 25.10 .2006 & \text { HPM } & 33 \text { anos } & \text { Espondilólise } & \text { L5 } \\ \text { I. L. M. } & 29.01 .2007 & \text { HPM } & 34 \text { anos } & \text { Espondilólise } & \text { L5 } \\ \text { A. N. A. } & 24.02 .2007 & \text { Santo lvo } & 16 \text { anos } & \text { Espondilolistese } & \text { L5 } \\ \text { L. G. M. } & 20.11 .2007 & \text { Baleia } & 11 \text { anos } & \text { Espondilolistese } & \text { L4 }\end{array}$

Apenas um desses pacientes praticava esporte competitivo em nível profissional. Os outros sete pacientes praticavam atividades esportivas e recreacionais nos finais de semana. Nenhum paciente apresentava dor tipo ciática. Um dos autores (BPCF) participou de todos os procedimentos cirúrgicos, com exceção de um caso, que foi realizado por equipe com o mesmo treinamento cirúrgico e seguindo a mesma padronização técnica. Todos os pacientes apresentavam imagem radiográfica de lesão da pars interarticularis. O local mais frequente de espondilólise envolvia o nível L5. Apenas em dois casos afetava o nível L4. Nenhum caso era unilateral.

Todos os pacientes foram submetidos a estudos por imagens com tomografia computadorizada (Figura 1), com cortes

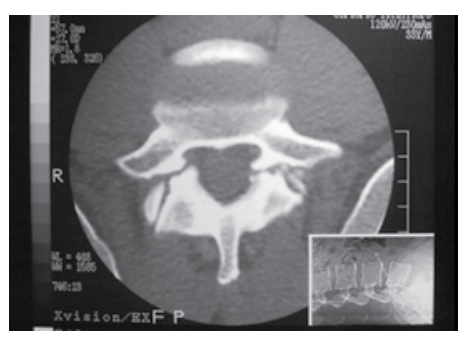

Figura 1

Avaliação por tomografia computadorizada multi-slice através da incidência especial Reverse Gantry (ver detalhe no canto direito inferior) foi escolhido para possibilitar os estudos por ressonância magnética, que podem ser necessários no futuro. O procedimento cirúrgico foi realizado por meio de pequena incisão cirúrgica mediana sobre o nível apropriado, com dissecção mínima para expor o defeito da pars interarticularis e permitir desbridamento do foco da pseudartrose, com exérese do material fibroso e decorticação das extremidades da fratura. Com uso de trépano elétrico de baixa rotação e sob controle radioscópico posicionam-se os fios guias, que têm ponto de entrada na base medial da faceta em direção ao defeito da pars interarticularis, para ancorarse na porção proximal do defeito, na junção entre o pedículo e o transverso. Durante a passagem do fio guia pode-se ter visão direta do mesmo pelo defeito da pars interarticularis, aumentando a segurança e precisão e melhorando a redução por compressão inter-fragmentar. Após posicionamento adequado dos fios guia, utiliza-se uma fresa de 2,5 mm canulada. Realizado o preparo do trajeto dos parafusos, é retirado pelo mesmo acesso, através do subcutâneo, com uso de trefinas ou osteótomos delicados, enxerto ósseo da crista ilíaca unilateralmente. Uma vez disponível o enxerto esponjoso, parte dele é colocado no defeito ósseo desbridado e então é realizada a passagem de parafuso esponjoso de titânio de 3,5mm parcialmente rosqueados sob controle radioscópico, certificandose que não existam roscas no arco neural, a fim de conferir adequada compressão inter-fragmentar da lesão. Depois de adequadamente reduzida e fixada a lesão, procede-se a decorticação superficial da região em torno do defeito, proximal e 
distalmente, e então é adicionado o restante do enxerto sobre a lesão. Uma vez conferida a redução do foco de fratura da pars e o posicionamente dos implantes e dos enxertos, realiza-se o fechamento da ferida por planos, sem a utilização de drenos. Em nenhum paciente foi realizado descompressão do canal espinhal (Figura 2).

No pós-operatório não foi utilizada órtese tóracolombossacral devido aos dados inconsistentes e conflitantes na literatura, ao incômodo desnecessário ao paciente e à imobilização comprovadamente insatisfatória para a junção lombossacral ${ }^{14,15}$.

O acompanhamento da consolidação foi feito com radiografias nas visitas de rotina pós-operatórias até a observação de fusão. Consideramos como fusão quando observamos osso trabecular atravessando o defeito. Foram utilizadas as incidências ântero-posteriores (AP), perfil e oblíquas. Nos casos em que não se observou fusão evidente até o período de seis meses, o acompanhamento passou a ser feito por tomografia computadoriza multi-slice até observação de consolidação do defeito ou definição por pseudartrose definitiva (Figura 3).

Os dados foram colhidos dos prontuários das instituições, nas quais foram realizadas as cirurgias, e todos os dados eram documentados sistematicamente, incluindo os critérios de Henderson (Quadro 2), a satisfação dos pacientes com o procedimento e se eles se submeteriam ao procedimento novamente. Foram colhidas também informações com relação às complicações de qualquer espécie.
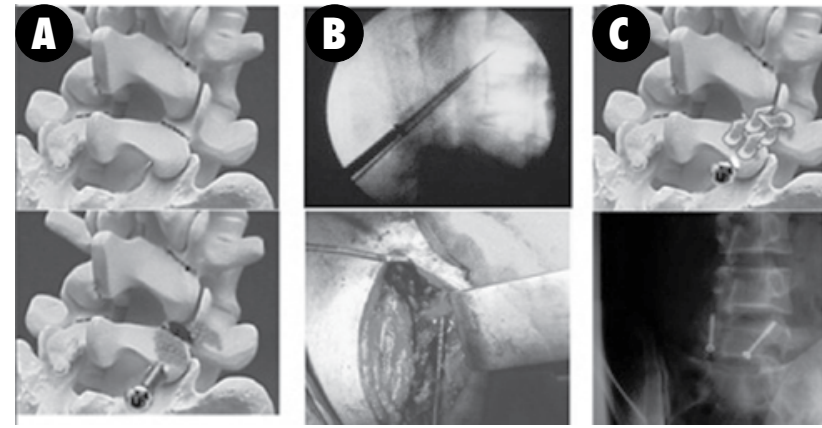

Figura 2

Técnica cirúrgica: A) Exposição e desbridamento do foco da espondilólise; B) Passagem de fio guia macheamento e passagem do parafuso sob visão direta e radioscópica; C) Fixação final e colocação de enxerto

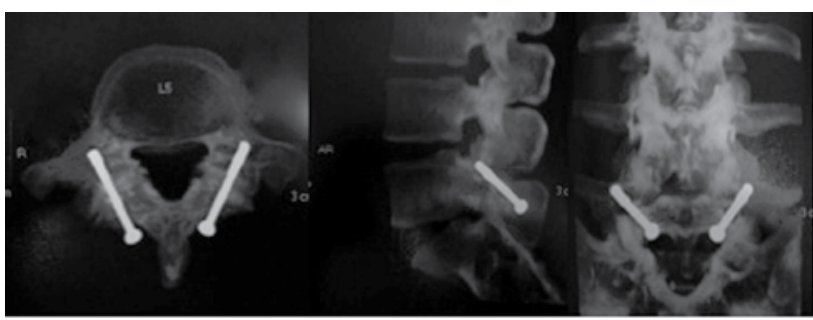

Figura 3

Controle de consolidação com tomografia computadorizada multi-slice

\section{QUADRO 2 - Critérios de Henderson ${ }^{31}$}

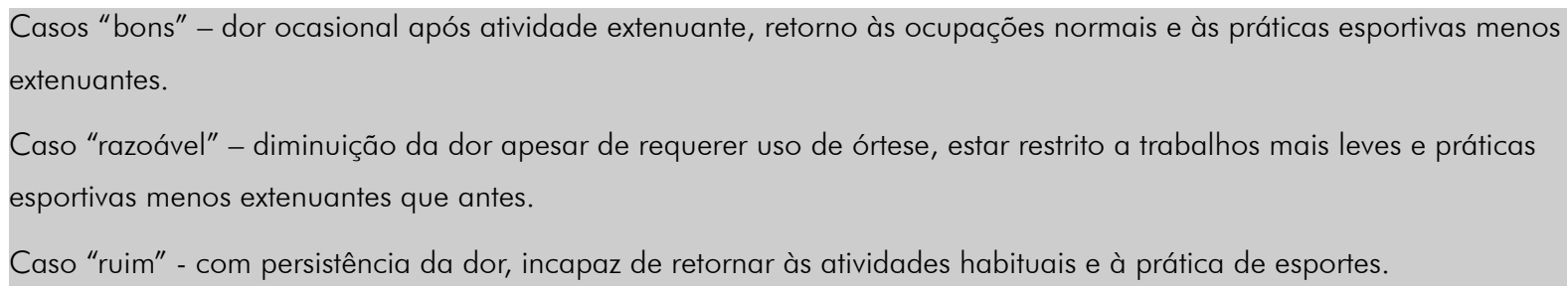

\section{RESULTADOS}

O resultado clínico avaliado de acordo com os critérios de Henderson demonstrou sete casos com excelentes resultados $(87,5 \%)$ e um caso de bom resultado $(12,5 \%)$ : nenhum paciente queixou-se de dor em crista ilíaca posterior (área doadora do enxerto); não foram constatadas infecções de ferida operatória ou profundas; em um caso foi observada discreta parestesia e paresia temporária da raiz de L5 à direita, causada por posicionamento medial do parafuso, que teve resolução espontânea sem a necessidade de reposicionamento do implante; não houve necessidade de transfusão de sangue em nenhum caso da amostra.

Em quase todos os casos, a consolidação da pars interarticularis foi visível com osso trabecular atravessando o defeito, com exceção de um caso, em que se observou pseudartrose $(12,5 \%)$, sendo que não houve quebra ou afrouxamento dos implantes. Esse caso foi classificado como bom resultado pelos critérios de
Henderson. Apesar de ter sido indicada a revisão cirúrgica, devido ao bom resultado clínico, o paciente e seus familiares optaram por não fazer nova intervenção, sendo mantido o acompanhamento clínico e radiológico, com o paciente mantendo-se sem intercorrências até o momento desse trabalho.

O tempo médio de acompanhamento desses pacientes foi de 23 meses, variando de seis a 43 meses. O tempo de consolidação óssea do defeito da pars interarticularis foi inferior a seis meses, com exceção de dois casos. Em um caso foi constatada consolidação à tomografia computadorizada multi-slice apenas no $9^{\circ}$ mês de pós-operatório e em outro houve definição como pseudartrose.

No momento da última avaliação registrada nos prontuários, todos os pacientes encontravam-se satisfeitos com os resultados da cirurgia e responderam que se submeteriam ao tratamento novamente (Quadro 3). 


\section{QUADRO 3 - Resultados da cirurgia e satisfação com o procedimento}

$\begin{array}{llllll}\text { Nome } & \text { Consolidação } & \begin{array}{l}\text { Tempo de } \\ \text { acompanhamento }\end{array} & \begin{array}{l}\text { Escala de } \\ \text { Henderson }\end{array} & \begin{array}{l}\text { Satisfação com } \\ \text { o procedimento }\end{array} & \begin{array}{l}\text { Realizaria novamente } \\ \text { o procedimento }\end{array} \\ \text { J. S. } & \text { Bilateral } & 43 \text { meses } & \text { Excelente } & \text { Sim } & \text { Sim } \\ \text { F. J. S. } & \text { Bilateral } & 34 \text { meses } & \text { Excelente } & \text { Sim } & \text { Sim } \\ \text { A. N. M. } & \text { Bilateral } & 28 \text { meses } & \text { Excelente } & \text { Sim } & \text { Sim } \\ \text { P. P. } & \text { Bilateral } & 22 \text { meses } & \text { Excelente } & \text { Sim } & \text { Sim } \\ \text { W. C. S. } & \text { Bilateral } & 20 \text { meses } & \text { Excelente } & \text { Sim } & \text { Sim } \\ \text { I. L. M. } & \text { Bilateral } & 16 \text { meses } & \text { Excelente } & \text { Sim } & \text { Sim } \\ \text { A. N. A. } & \text { Pseudartrose bilateral } & 15 \text { meses } & \text { Bom } & \text { Sim } & \text { Sim } \\ \text { L. G. M. } & \text { Bilateral } & 6 \text { meses } & \text { Excelente } & \text { Sim } & \text { Sim }\end{array}$

\section{DISCUSSÃO}

As técnicas de reconstrução do defeito na pars interarticularis emergiram como opção para a realização da artrodese, no manejo dos casos de dor lombar por espondilólise refratária ao tratamento conservador, por serem técnicas que preservam a anatomia lombar, a mobilidade do segmento e por serem procedimentos menos invasivos. Os princípios do tratamento cirúrgico do reparo direto da espondilólise são os mesmos usados para o tratamento de pseudartrose de um osso longo: desbridamento, colocação de enxerto ósseo no foco da pseudartrose e compressão por meio da fratura ${ }^{16}$.

A técnica de Buck demonstra-se superior, em vários aspectos, aos demais métodos, como o reparo pela técnica de Scott ou Scott modificada, nas quais se exige maior exposição cirúrgica e apresentam maior índice de falha dos implantes ${ }^{17}$. A falha dos implantes também é um ponto fraco da técnica de Morscher ${ }^{18}$, assim como seus maus resultados clínicos em pacientes com idade superior a $17 \operatorname{anos}^{19}$. As técnicas que utilizam ganchos, em linhas gerais, apresentam a desvantagem de usar implantes mais salientes e a necessidade de maior exposição cirúrgica ${ }^{20}$. Altas taxas de sucesso têm sido empregadas com várias técnicas de fixação ${ }^{4}$. Do ponto de vista biomecânico, o reparo usando parafuso atravessando a espondilólise tem maior força e rigidez. As técnicas com amarrilho são as construções menos estáveis quando testadas contra cargas de flexão e, portanto, têm menor probabilidade de que o novo osso atravesse o defeito ${ }^{17}$. As técnicas que envolvem o uso de parafusos pediculares também têm sido criticadas em virtude do risco de violarem o espaço discal superior e culminarem, subseqüentemente, em aumento do movimento nesse segmento já vulnerável pela lesão da pars interarticularis (também suscetível a discopatia) ${ }^{21}$ (Figura 4).
O reparo aberto foi inicialmente descrito por Buck, em 1970, utilizando parafusos AO de 4,5mm de aço inoxidável para estabilizar e comprimir os fragmentos de fratura ${ }^{22}$. Os bons resultados dessa técnica continuam ainda hoje sendo divulgados na literatura, inclusive para o tratamento de lesões unilaterais ${ }^{10,23,24}$. Melhora significativa da dor e da função têm sido documentadas mesmo na ausência de consolidação do defeito $^{15,25}$. Várias séries publicam que até $90 \%$ dos atletas profissionais retornam ao mesmo nível de atividade em meses ${ }^{10,23}$. Os resultados obtidos estão de acordo com aqueles encontrados na literatura. Nessa pesquisa foi apresentado apenas um atleta profissional, o qual retornou às atividades no mesmo nível prévio ao quadro álgico, inclusive, participando da primeira divisão do Campeonato Brasileiro de Futebol, como goleiro de um dos grandes time do país. Entre os pacientes também constavam três policiais militares, que retornaram às atividades operacionais, que exigem uso de armamentos e materiais de proteção pessoal pesados, sem limitações.

Críticas à técnica de Buck estão ligadas ao risco de complicações neurológicas ou mecânicas e à dificuldade técnica ${ }^{22,16}$. Mas não foi constatada nenhuma complicação neurológica definitiva ou mecânica. Acredita-se que a explicação esteja relacionada a fatores como o conhecimento detalhado da anatomia da pars interarticularis e domínio da técnica de compressão do foco da pseudartrose por meio do uso do parafuso ${ }^{26}$.

A idade era considerada fator limitante para o bom resultado da reconstrução ístmica ${ }^{27}$. Observou-se, entretanto, que o grau de degeneração discal nos níveis adjacentes é mais determinante para a indicação de artrodese do que a idade do paciente ou o grau de listese ${ }^{13}$. Por isso, o estudo pré-operatório com ressonância magnética assume função crucial na avaliação dos discos. A propedêutica pode ser aprofundada com injeções diagnósticas ${ }^{23}$. Discografia provocativa pode ser elucidativa na investigação de dor discogênica proveniente de discos
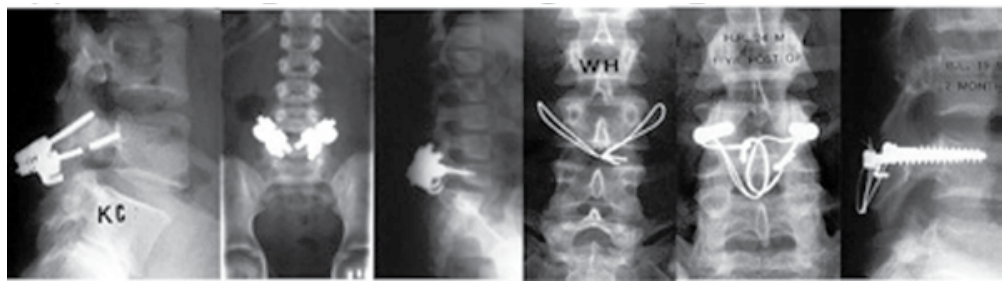

Figura 4

Complicações de outras técnicas de reconstrução ístmica: A) Quebra de instrumentais pela técnica de Morsher; B) Volume do instrumental utilizado com ganchos e parafusos; C) Fragilidade da fixação com fios; D) Agressão tecidual desnecessária pela técnica de Scott modificada 
degenerados ${ }^{28}$. Apesar desses conceitos serem aceitos e adotados na maior parte dos estudos que envolvem reconstrução ístmica, existem trabalhos em que o uso da técnica é expandido a pacientes com idade mais avançada que a média e com evidências de degeneração discal, com resultados igualmente satisfatórios ${ }^{29}$.

Os estudos experimentais afirmam que o reparo da espondilólise diminui a hiper-mobilidade aproximando-a de valores mais próximos dos fisiológicos ${ }^{5,21}$. Isso pode ser traduzido clinicamente com a publicação de Rafig et al., que constata o efeito protetor do reparo sobre os discos adjacentes em uma revisão a longo prazo $^{30}$.

\section{CONCLUSÃO}

O reparo direto da lesão da pars interarticularis pela técnica de Buck modificada pelos autores demonstra ser eficaz, clínica e radiologicamente, para o tratamento de espondilólise e espondilolistese grau I, em pacientes com lombalgia que não respondem ao tratamento conservador. $\mathrm{O}$ método demonstra ser seguro, com baixas taxas de complicações e, por ser minimamente invasivo, agride pouco a musculatura e as articulações da coluna lombar, não sendo necessário em nenhum caso o uso de hemoderivados ou órteses.

\section{Agradecimento}

Ao Dr. Ujiwal K. Debnath, por sua visita a Belo Horizonte, quando nos ensinou a técnica de Buck inspirando-nos com sua experiência e disponibilidade.

\section{REFERÊNCIAS}

1. Standaert CJ, Herring SA. Expert opinion and controversies in sports and musculoskeletal medicine: the diagnosis and treatment of spondylolysis in adolescent athletes. Arch Phys Med Rehabil. 2007;88(4):537-40.

2. Herkowitz HN, Garfin SR, Eismont FJ, Bell GR, Balderston RA. Rothman-Simeone The Spine. In: Parke WW, Bono CM, Garfin SR. Applied anatomy of the spine. Philadelphia: Saunders; 2006. p. 16-17.

3. Szypryt EP, Twining P, Mulholland RC, Worthington BS. The prevalence of disc degeneration associated with neural arch defects of the lumbar spine assessed by magnetic resonance imaging. Spine. 1989;14(9):977-81.

4. Vathana P, Prasartritha T. A biomechanic study of the surgical repair technique of pars defect in spondylolysis. J Med Assoc Thai. 1998;81(11):824-9.

5. Sairyo K, Goel VK, Faizan A, Vadapalli S, Biyani S, Ebraheim N. Buck's direct repair of lumbar spondylolysis restores disc stresses at the involved and adjacent levels. Clin Biomech (Bristol, Avon). 2006;21(10):1020-6.

6. Pizzutillo PD, Hummer CD 3rd. Nonoperative treatment for painful adolescent spondylolysis or spondylolisthesis. J Pediatr Orthop. 1989;9(5):538-40.

7. Jacobs WC, Vreeling A, De Kleuver M. Fusion for low-grade adult isthmic spondylolisthesis: a systematic review of the literature. Eur Spine J. 2006;15(4):391-402.

8. Sherman FC, Rosenthal RK, Hall JE. Spine fusion for spondylolysis and spondylolisthesis in children. Spine. 1979;4(1):59-66.

9. Pedersen AK, Hagen R. Spondylolysis and spondylolisthesis. Treatment by internal fixation and bone-grafting of the defect. J Bone Joint Surg Am. 1988;70(1):15-24.

10. Debnath UK, Freeman BJ, Gregory P, de la Harpe D, Kerslake RW, Webb JK. Clinical outcome and return to sport after the surgical treatment of spondylolysis in young athletes. J Bone Joint Surg Br. 2003;85(2):244-9.
11. Saifuddin A, White J, Tucker S, Taylor BA. Orientation of lumbar pars defects: implications for radiological detection and surgical management. J Bone Joint Surg Br. 1998;80(2):208-11.

12. Delgado DS, Daniel MM, Leite CC. Interposição da gordura epidural entre a dura-máter e o processo espinhoso: um sinal para o diagnóstico de espondilólise pela ressonância magnética da coluna lombar. Coluna/ Columna. 2006;6(2):57-60.

13. Dai LY. Disc degeneration in patients with lumbar spondylolysis. J Spinal Disord. 2000;13(6):478-86.

14. Ruiz-Cotorro A, Balius-Matas R, EstruchMassana AE, Vilaró Angulo J. Spondylolysis in young tennis players. Br J Sports Med. 2006;40(5):441-6.

15. Standaert CJ, Herring SA. Spondylolysis: a critical review. Br J Sports Med. 2000;34(6):415-22.

16. Freeman B. Scoliosis and Kyphosis. In: Canale ST. Campbell's operative orthopaedics. Philadelphia: Mosby; 2003. p. 1896.

17. Kip PC, Esses SI, Doherty BI, Alexander JW, Crawford MJ. Biomechanical testing of pars defect repairs. Spine. 1994;19(23):2692-7.

18. Dreyzin V, Esses SI. A comparative analysis of spondylolysis repair. Spine. 1994;19(17):1909-14.

19. Winter M, Jani L. Results of screw osteosynthesis in spondylolysis and low-grade spondylolisthesis. Arch Orthop Trauma Surg. 1989;108(2):96-9.

20. Tokuhashi Y, Matsuzaki H. Repair of defects in spondylolysis by segmental pedicular screw hook fixation. A preliminary report. Spine. 1996;21(17):2041-5.

21. Mihara H, Onari K, Cheng BC, David SM, Zdeblick TA. The biomechanical effects of spondylolysis and its treatment. Spine. 2003;28(3):235-8.

22. Buck JE. Direct repair of the defect in spondylolisthesis. Preliminary report. J Bone Joint Surg Br. 1970;52(3):432-7.

23. Suh PB, Esses SI, Kostuik JP. Repair of pars interarticularis defect. The prognostic value of pars infiltration. Spine. 1991;16(8 Suppl):S445-8.
24. Debnath UK, Freeman BJ, Grevitt MP, Sithole J, Scammell BE, Webb JK. Clinical outcome of symptomatic unilateral stress injuries of the lumbar pars interarticularis. Spine. 2007;32(9):995-1000.

25. Toribio JA, Pellise F, Garcia-Fontecha C, Bago J, Villanueva C. Clinical and CT evaluation of direct defect repair in spondylolysis. J Bone Joint Surg Br. 1998;80 (Suppl 3):232.

26. Perren SM, Frigg R, Hehli M, Tepic S. Parafuso de tração. In: Rüedi TP, Murphy WM. Princípios ao do tratamento de fraturas. Porto Alegre: Artmed; 2002. p.157-67.

27. Roca J, Ubierna MT, Iborra M, CavanillesWalker JM. Spondylolysis repair - clinical and $\mathrm{CT}$ assessed study. J Bone Joint Surg Br. 2001;83 (Suppl 2):220.

28. Wu SS, Lee CH, Chen PQ. Operative repair of symptomatic spondylolysis following a positive response to diagnostic pars injection. J Spinal Disord. 1999;12(1):10-6.

29. Dai LY, Jia LS, Yuan W, Ni B, Zhu HB. Direct repair of defect in lumbar spondylolysis and mild isthmic spondylolisthesis by bone grafting, with or without facet joint fusion. Eur Spine J. 2001;10(1):78-83.

30. Rafig M, Marks DS, Thompson AG. MRI findings in the intervertebral disc following previous modified scott repair for spondylolysis. Bone Joint Surg Br. 2003;85 (Suppl 3):192.

31. Henderson ED. Results of the surgical treatment of spondylolisthesis. J Bone Joint Surg Am. 1966;48(4):619-42.

\section{Correspondência}

Bruno Pinto Coelho Fontes

Rua Bernardo Guimarães, 1444

/ Lourdes

CEP: 30140-081

Belo Horizonte (MG), Brasil.

E-mail: brunopcfontes@yahoo.com.br 